Diabetologia (1994) 37:286-292

\title{
Glycosaminoglycans in the human aorta in diabetes mellitus: a study of tunica media from areas with and without atherosclerotic plaque
}

\author{
L. Heickendorff ${ }^{1}$, T. Ledet ${ }^{2}$, L.M. Rasmussen ${ }^{2}$ \\ ${ }^{1}$ Department of Clinical Chemistry, Aarhus University Hospital, Amtssygehuset, Aarhus, Denmark \\ ${ }^{2}$ Research Laboratory for Biochemical Pathology, University Institute of Pathology, Kommunehospitalet, University of Aarhus, \\ Denmark
}

Summary Alterations in the connective tissue of the arterial wall have been suggested to play a role in the development of macrovascular disease in diabetes mellitus. The present study deals with changes in the content of GAG in aortic tunica media in human diabetes by separately analysing normal areas and areas with fibrous plaques. The thoracic aorta from 15 diabetic patients ( 7 with IDDM, 8 with NIDDM), and 30 sex- and age-matched non-diabetic subjects were collected at autopsy. Tunica intima was removed and GAG were isolated from the dried defatted and pulverized tunica media. GAG were quantified by uronic acid analysis and characterized by electrophoresis on cellulose acetate. Results showed that IDDM patients had a relative and absolute increase in hyaluronic acid in normal tunica media compared to non-diabetic subjects. There was a significant positive correlation between hyaluronic acid content of normal tunica media and duration of diabetes, but not between hyaluronic acid content and age. When tunica media from plaque areas was compared to normal areas the same pattern was evident in diabetic patients as in non-diabetic patients - significantly increased proportion of dermatan sulphate and reduced hyaluronic acid. The data agree with the notion that the arterial wall is subject to different pathological processes in diabetes, one of classical atherosclerosis with changes in GAG similar to non-diabetic subjects, and the other seen in areas without plaques with dissimilar alterations in GAG. These data therefore support the concept of the presence of a macrovascular disease in diabetes different from atherosclerosis. [Diabetologia (1994) 37:286-292]

Key words Diabetes mellitus, macrovascular disease, atherosclerosis, aorta, tunica media, glycosaminoglycans, hyaluronic acid, heparan sulphate.
Diabetic patients are at risk of developing macrovascular disease, however, the nature of and pathogenetic mechanisms behind the large vessel disease is only poorly understood. Present knowledge about structural and biochemical alterations of the connective tissue in the arterial wall in diabetes mellitus indicates that

Received: 7 June 1993

and in revised form: 22 September 1993

Corresponding author: Dr. L. Heickendorff, Department of Clinical Chemistry, Aarhus University Hospital, Amtssygehuset Tage Hansensgade 2, DK-8000 Aarhus C, Denmark

Abbreviations: NIDDM, Non-insulin-dependent diabetes mellitus; IDDM, insulin-dependent diabetes mellitus; GAG, glycosaminoglycans the large vessel disease cannot be explained solely as accelerated atherosclerosis [1]. Several investigations point toward primary alterations in the arterial wall in the diabetic patients. Proliferation of smooth muscle cells with increased periodic acid-Schiff positive substance in the extracellular matrix in the coronary arteries $[2-5]$ and linear arterial calcification in tunica media $[6,7]$ have been described in diabetes. In aorta from diabetic subjects fibronectin and type IV collagen accumulate in tunica media independent of the presence of atherosclerotic plaque $[8,9]$ and non-atherosclerotic areas of aorta have shown altered mechanical properties with significantly increased stiffness [10] indicating that alterations occur in the connective tissue of the arterial wall in diabetes.

It has been suggested that changes in the content or distribution of GAG in the arterial wall may occur in 
Table 1. Clinical data from IDDM and NIDDM patient groups and two groups of sex- and age-matched non-diabetic subjects

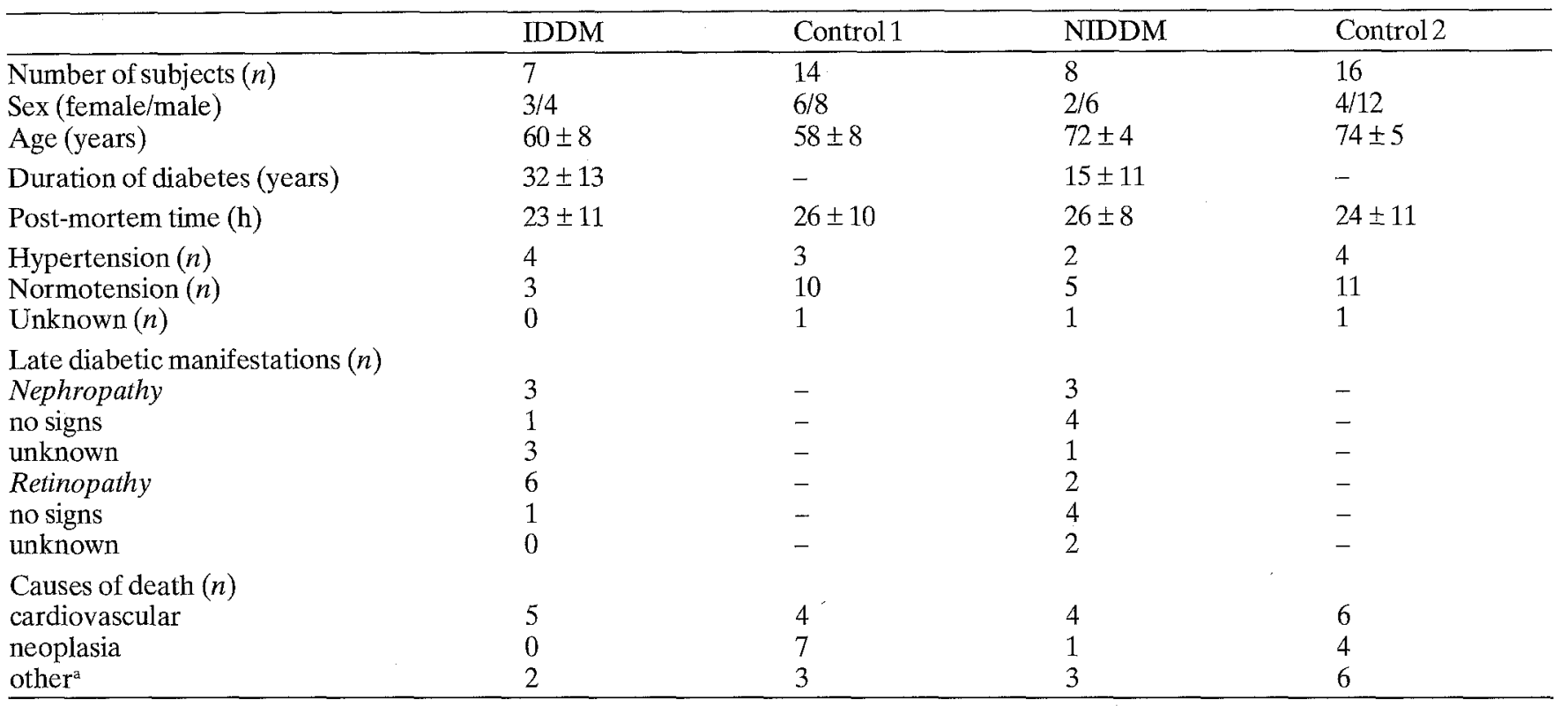

Values are presented as mean \pm SD. ${ }^{\text {a }}$ Infections, accidents etc.

diabetes. Morphological studies of diabetic arteries have shown decreased Alcian blue staining suggesting either a decreased GAG content or altered sulphation of the macromolecules [3]. Data from some studies of experimental diabetes have pointed to an increased hyaluronic acid content in rodent aorta $[11,12]$, others have given less clear results [13], however, there is no information available about the distribution of GAG in tunica media of the human aorta in diabetes.

GAG/proteoglycans are considered to be involved in some of the key events which lead to the formation of atherosclerotic lesions [14], e.g. binding of lipoproteins $[15,16]$, platelet aggregation [17], and growth inhibition of smooth muscle cells [18]. Alterations in these macromolecules in diabetes could therefore play a significant role in the development of large vessel disease in diabetes.

This study presents the first information about the content and distribution of GAG in tunica media of the human aorta in diabetes analysing separately tunica media from areas with and without atherosclerotic plaque.

\section{Subjects and methods}

\section{Patients}

The descending part of the thoracic aorta was collected at autopsy from 15 diabetic patients: seven with IDDM and eight with NIDDM. For each diabetic patient two sex and age-matched non-diabetic subjects were included. The aortic samples were in all cases obtained within $40 \mathrm{~h}$ of death. Exclusion criteria were: connective tissue diseases, glucocorticoid treatment, administration of chemotherapeutics or radiotherapy to the thoracic region. Basal clinical data of diabetic patients and control sub- jects are given in Table 1. Patients treated for hypertension or with more than one blood pressure measurement over 140/ $100 \mathrm{~mm} \mathrm{Hg}$ were considered to be hypertensive.

\section{Aortas}

The descending part of the thoracic aorta was removed and washed in $0.9 \% \mathrm{NaCl}, 10 \mathrm{mmol} / / \mathrm{Tris} / \mathrm{HCl}(\mathrm{pH}$ 7.4) containing proteinase inhibitors $(0.5 \mathrm{mmol} / \mathrm{l}$-ethyl-maleinide, $0.5 \mathrm{mmol} / \mathrm{l}$ phenylmethylsulphonyl fluoride, $1 \mathrm{mmol} / \mathrm{l}$ benzamidine hydrochloride) and kept at $-80^{\circ} \mathrm{C}$ until analysis. After thawing tunica adventitia was removed and intima-media samples were taken from areas with apparently normal intimal surface as well as from areas with plaque seen as yellowish/white elevated lesions. Normal intima was defined as areas without any discolorations or elevation of the surface, whereas regions with elevated white areas without ulceration, aneurysms or thrombosis were considered as uncomplicated fibrous plaque. Tunica intima was carefully removed from tunica media by blunt dissection. Histological examination confirmed that the tissue was split close to the internal elastic membrane. The samples were lyophilized, weighed, and defatted by two 24-h extractions in chloroformethanol (3:1). The samples were lyophilized and weighed. The dry defatted tissue was pulverized in a Retsch Mill (F.Kurt Retsch GMBH, Haan, Germany), and stored at $-80^{\circ} \mathrm{C}$ until analysis.

\section{Isolation of $G A G$}

The GAG were isolated from $10-30 \mathrm{mg}$ of dry defatted tissue as described in detail previously [19]. Tunica media samples were digested with papain, $5 \mu \mathrm{g} / \mathrm{mg}$ dry defatted tissue for $20 \mathrm{~h}$ at $65^{\circ} \mathrm{C}$. GAG were precipitated with cetyl pyridinium chloride at a final concentration of $0.5 \%$. Cetyl pyridinium chloride-GAG complexes were dissolved in n-propanol and further purified by precipitation twice with ethanol containing $2 \%$ potassium acetate. GAG were dissolved in distilled water and quantified on 
the basis of uronic acid content [20]. The efficacy of the proteolytic digestion procedure was evaluated by measuring the hexosamine content of the insoluble precipitate of the samples [21]. The data showed that $95-97 \%$ of the GAG were liberated by the present procedure. Recovery was estimated by adding known amounts of purified GAG to samples of dry defatted tissue, and was found to be $90-97 \%$. The results were not corrected for recovery.

\section{Identification of $G A G$}

Isolated GAG were separated by electrophoresis on cellulose acetate (Titan III, Helena Laboratories, Beaumont, Tx., USA). The samples were analysed in triplicate using two different buffer systems; a) electrophoresis in $0.3 \mathrm{~mol} / \mathrm{l}$ cadmium acetate, $\mathrm{pH} 4.1$ [22], and b) electrophoresis in $0.1 \mathrm{~mol} / \mathrm{l}$ barium acetate, pH 5.0 [23]. In cadmium acetate hyaluronic acid was well separated from heparan sulphate and dermatan/ chondroitin sulphates, whereas the chondroitin sulphate fraction was determined using the barium acetate buffer system. After staining in $0.1 \%$ Alcian blue the distribution of GAG was quantified by densitometry using a Shimazdu scanning spectrophotometer at $610 \mathrm{~nm}$, beam size $0.05 \times 2 \mathrm{~mm}$. The average variation (between runs) of the electrophoretic analysis of samples was $2-6 \%$. On each membrane a mixture of standards was co-electrophoresed. The following GAG were used as standards: hyaluronic acid from human umbilical cord, chondroitin sulphate $A$ from bovine trachea, chondroitin sulphate $\mathrm{C}$ from shark cartilage, dermatan sulphate from porcine skin, heparan sulphate from bovine kidney, all obtained from Sigma (St. Louis, Mo., USA). The difference in Alcian blue binding capacity of the various GAG was determined and the data were compensated accordingly. Relative to hyaluronic acid the following factors were used: chondroitin sulphate 0.6 , dermatan sulphate 0.6 , heparan sulphate 0.8 . The distribution/content of the GAG types was expressed as a fraction of total GAG (\%) and as $\mu \mathrm{g}$ GAG per $\mathrm{mg}$ dry defatted tissue.

\section{Statistical analysis}

Statistical evaluation was performed by non-parametrical analyses using the Wilcoxon test. Comparisons between variables were made using Spearman's Rank Correlation analysis. A $p$ value of less than 0.05 was considered significant.

\section{Results}

The clinical characteristics of the two diabetic patient groups and the non-diabetic control groups are given in Table 1. There was no statistically significant difference in time between death and examination in either of the groups examined.

\section{Diabetic groups compared to non-diabetic groups}

Total GAG content of tunica media. Patients with NIDDM had significantly reduced amounts of total GAG in tunica media from non-plaque areas when compared to a group of age- and sex-matched non-

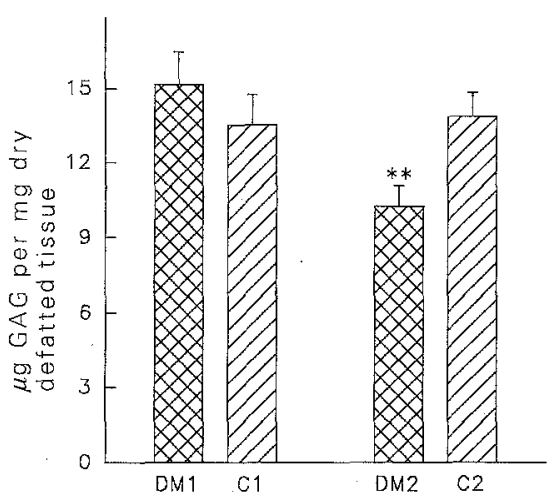

Fig. 1. Content of total GAG in tunica media from areas of aorta with macroscopically normal intima. Mean values of $\mu \mathrm{g} G \mathrm{GG}$ per $m g$ dry defatted tissue \pm SEM are shown for seven IDDM subjects (DM1), eight NIDDM patients (DM2), and two groups of non-diabetic subjects matched for age and $\operatorname{sex}(\mathrm{C} 1, n=14$; and $\mathrm{C} 2, n=16$ ). $* * 2 p<0.01$

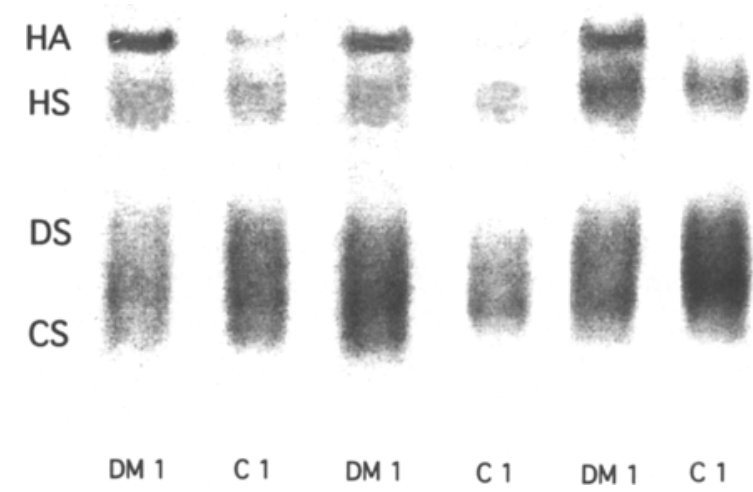

Fig.2. Electrophoresis on cellulose acetate of GAG isolated from tunica media of non-atherosclerotic areas of aorta. Samples from three IDDM patients (DM1) and non-diabetic subjects (C1) are shown. Electrophoresis was performed in $0.3 \mathrm{~mol} / \mathrm{l}$ cadmium acetate, $\mathrm{pH} 4.1$ to separate hyaluronic acid (HA) from heparan sulphate and dermatan sulphate/chondroitin sulphates (DS and CS)

diabetic subjects (Fig.1). We found no difference between the group of IDDM patients and the control group. Samples of tunica media from plaque areas from both groups of diabetic patients had a similar content of total GAG as the control groups (not shown).

Distribution of $G A G$ in tunica media from areas without plaque. Figure 2 shows a typical electrophoretic pattern of GAG on cellulose acetate from tunica media of aorta from IDDM patients and control subjects. Increased density of the hyaluronic acid band is apparent in samples from the diabetic patients. Hyaluronic acid constituted in average $17.6 \%$ of total GAG in tunica media from normal areas of aorta from the IDDM patient group, but only $10.1 \%$ in the non-diabetic control group (Fig. 3). This difference was highly significant $(2 p<0.01)$. In the NIDDM 

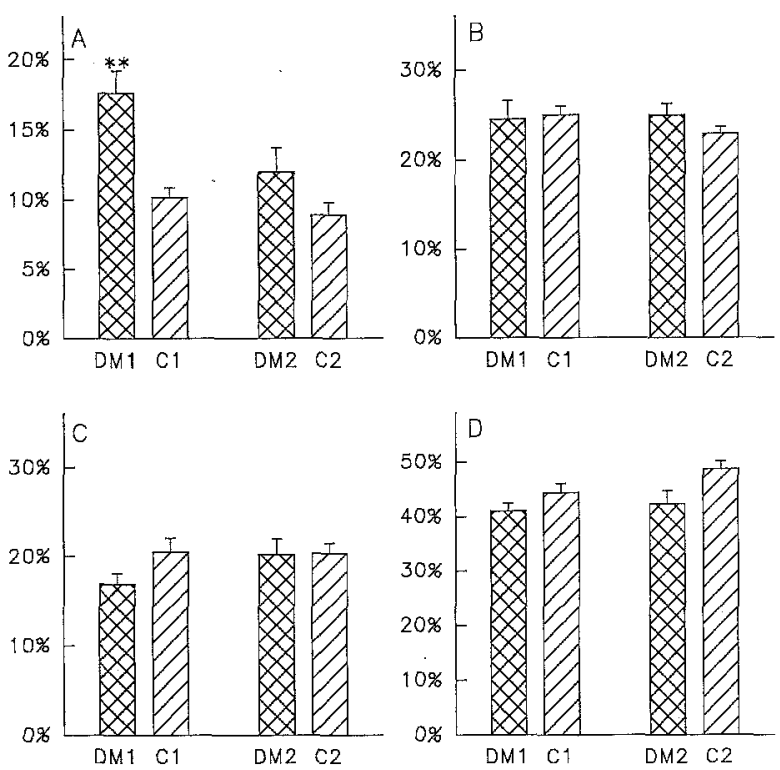

Fig.3A-D. Proportions of hyaluronic acid (A), heparan sulphate (B), dermatan sulphate $(\mathbf{C})$ and chondroitin sulphates $(\mathbf{D})$ in tunica media from areas of aorta with macroscopically normal intima. Mean percentages of total GAG \pm SEM are given for seven IDDM patients (DM1), eight NIDDM patients (DM2) and two groups of non-diabetic subjects matched for age and $\operatorname{sex}(\mathrm{C} 1, n=14$; and $\mathrm{C} 2, n=16)$. $* * 2 p<0.01$

patient group there was a trend towards increased hyaluronic acid fraction in tunica media from nonatherosclerotic areas of the vessel, but the difference failed to meet statistical significance $(12.0 \%$ hyaluronic acid vs $8.9 \%$, mean values, $0.05<2 p$ $<0.10)$.

We found no statistically significant differences in the proportion of heparan sulphate, dermatan sulphate or chondroitin sulphate between the diabetic patients and their control groups in samples of tunica media from non-atherosclerotic areas of aorta (Fig.3).

When the results were calculated in absolute terms on the basis of uronic acid content, the IDDM patient group had an increased amount of hyaluronic acid per $\mathrm{mg}$ dry defatted tissue (mean $\mu \mathrm{g}$ hyaluronic acid per $\mathrm{mg}$ dry defatted tissue: $2.67 \mu \mathrm{g}$ vs $1.41 \mu \mathrm{g}, 2 p<0.01$, not shown). We found no statistically significant difference in heparan sulphate content, dermatan sulphate or chondroitin sulphate per mg dry defatted tissue when comparing the diabetic patient groups to the control groups.

Distribution of $G A G$ in tunica media from areas with plaque. When samples of tunica media from areas with plaques were examined the proportion of the various GAG types in both IDDM and NIDDM patient groups was similar to that of the control groups (Fig. 4).

$G A G$ and duration of diabetes. The relationship between the duration of diabetes of all diabetic patients and hyaluronic acid content or hyaluronic acid fraction
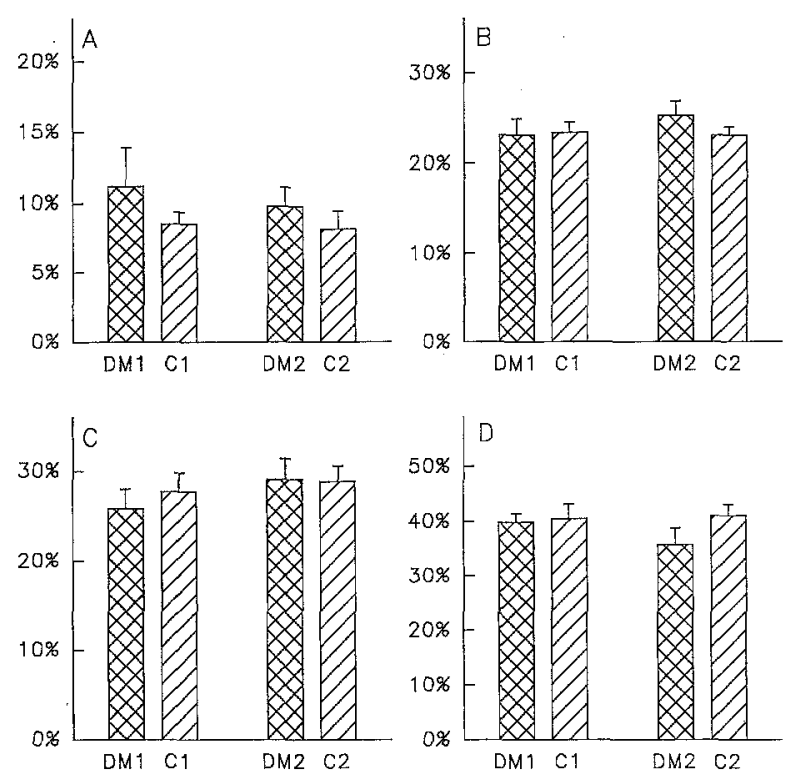

Fig.4A-D. Proportions of hyaluronic acid (A), heparan sulphate (B), dermatan sulphate (C) and chondroitin sulphates $(\mathbf{D})$ in tunica media from areas of aorta with plaque. Mean percentages of total $G A G \pm S E M$ are given for seven IDDM patients (DM1), eight NIDDM patients (DM2) and two groups of non-diabetic subjects matched for age and sex (C1, $n=14$; and $\mathrm{C} 2, n=16$ ).

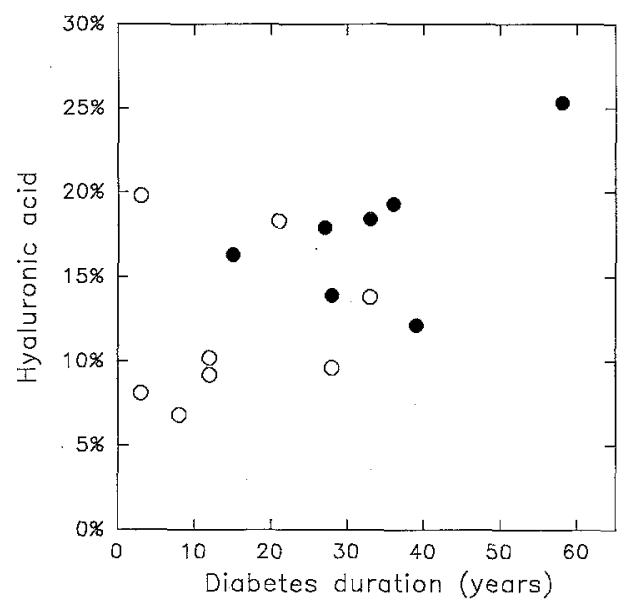

Fig.5. The relationship between duration of diabetes and proportion of hyaluronic acid (\% of total GAG) in tunica media of aorta from 15 diabetic subjects. Samples were taken for analysis of GAG from areas with macroscopically normal intima. $p=0.05, r=0.47$. , seven IDDM patients; $O$, eight NIDDM patients

of tunica media from non-atherosclerotic areas of aorta was evaluated. We found a significant positive association between duration of diabetes and hyaluronic acid content $(r=0.49, p=0.03, n=15)$ and hyaluronic acid fraction $(r=0.47, p=0.05, n=15)$ as shown in Figure 5. There was a trend towards a positive relationship between GAG content and diabetes duration $(r=0.42$, $p=0.08, n=15)$ and a negative association between GAG content and age $(r=-0.41, p=0.06, n=15)$. The 
relationship between age and hyaluronic acid fraction or hyaluronic acid content was statistically insignificant $(r=-0.20, p=0.25$ and $r=-0.35, p=0.13$, respectively).

\section{Tunica media from plaque areas compared to non-plaque areas}

The total amount of GAG from tunica media of plaque areas was significantly decreased compared to normal areas of aorta. The difference was significant in both the combined non-diabetic groups $(2 p<0.01)$ and in the IDDM group $(2 p<0.01)$, but not in the NIDDM patient group. The following mean values of $\mu \mathrm{g}$ GAG per $\mathrm{mg}$ dry defatted tissue were found in nonplaque/plaque areas: IDDM group, $15.2 \mu \mathrm{g} / 10.4 \mu \mathrm{g}$; NIDDM group, $10.3 \mu \mathrm{g} / 8.6 \mu \mathrm{g}$; non-diabetic groups combined, $13.8 \mu \mathrm{g} / 9.5 \mu \mathrm{g}$.

When the proportion of the various GAG in tunica media was analysed comparing plaque areas to normal areas the most consistent findings were increased dermatan sulphate and decreased hyaluronic acid fractions. Tunica media from plaque areas of aorta had a significantly increased dermatan sulphate fraction. This was true both for IDDM and NIDDM patients as well as for the non-diabetic subjects (IDDM patients: $2 p<0.01$, NIDDM patients: $2 p<0.05$, and non-diabetic subjects: $2 p<0.01$ ) (Fig. 6 ). In tunica media of aorta from IDDM patients we found a significantly decreased proportion of hyaluronic acid in plaque areas of the vessel compared to macroscopically normal areas $(2 p<0.05)$. Also expressed per mg dry defatted tissue the hyaluronic acid content was less in tunica media samples from areas with plaque in IDDM patients $(2 p<0.02)$. The same pattern was seen in nondiabetic subjects, as we observed decreased amounts of hyaluronic acid in tunica media from plaque areas of aorta $(2 p<0.01)$. In NIDDM patients the difference in hyaluronic acid fraction in tunica media between plaque areas and normal areas of aorta was not statistically significant.

\section{Discussion}

Previous reports have shown alterations in several extracellular matrix components, such as accumulation of fibronectin and type IV collagen in arteries from diabetic patients $[8,9]$. In the present study which focuses on another class of extracellular matrix components, GAG, we found increased hyaluronic acid content in aortas from diabetic patients. This result agrees with earlier studies of experimental diabetes performed on alloxan-diabetic rodents where it was observed that hyaluronic acid accumulated in tunica media-intima samples from the aorta of diabetic animals, without any changes in total GAG content or
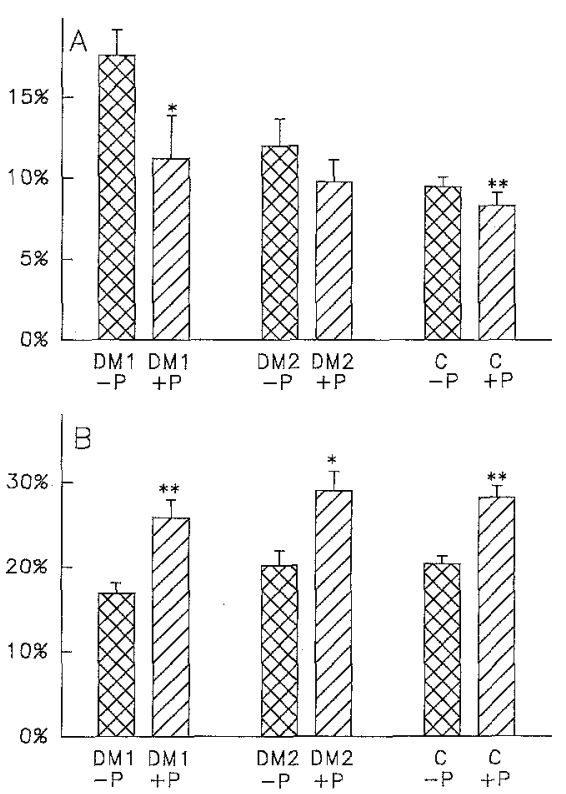

Fig. 6A,B. Comparison between tunica media from areas of aorta without plaque $(-\mathrm{P})$ and with plaque $(+\mathrm{P})$. Proportions of hyaluronic acid (A) and dermatan sulphate (B) in tunica media of aorta from seven IDDM patients (DM1), eight NIDDM patients (DM2) and 30 non-diabetic subjects (C) are shown. Data are given as percentages of total GAG, mean values \pm SEM. $* * 2 p<0.01$. $* 2 p<0.05$

concentration of heparan sulphate [11, 12]. Hyaluronic acid is secreted by arterial smooth muscle cells [24], and it is interesting to note that diabetic serum enhances the secretion of hyaluronic acid from cultured human aortic smooth muscle cells $[25,26]$. Factors in the diabetic metabolism may stimulate hyaluronic acid synthesis leading to tissue accumulation of this substance in the tunica media of arteries, as we report here. In support of this view is the fact that we could demonstrate a statistically significant relationship between hyaluronic acid content in tunica media and duration of diabetes. This relationship did not reflect influence of age, since hyaluronic acid concentration did not show a positive correlation to age in either diabetic or control subjects.

The accumulation of hyaluronic acid in tunica media of aorta could also be related to increased growth rates of vascular smooth muscle cells in diabetes which has been suggested [4, 27,28]. Previous studies of arterial smooth muscle cells in vitro have shown that the cells increase their activity of hyaluronic acid synthesizing enzymes during proliferation [29], and that growth-stimulated cells increase the secretion of hyaluronic acid [30]. Studies with other cell types support this notion, since it has been reported that hyaluronic acid synthesis is related to cell proliferation in fibroblasts [31], and that hyaluronic acid synthesis may be regulated by growth regulating factors, such as transforming growth factor $\beta$ and plateletderived growth factor $[32,33]$. 
Data from in vitro labelling techniques have suggested that aortic tissue from diabetic animals display decreased synthesis of heparan sulphate and also other sulphated GAG [11, 34]. A reduction in heparan sulphate content has been demonstrated in the kidney/glomerular basement membranes in diabetes [35, 36]. However, we did not find any reduction in heparan sulphate content of tunica media in the diabetic aorta, either when expressed in absolute amount per mg dry defatted tissue or as a percentage of total GAG. This observation indicates that the decrease in heparan sulphate content seen in glomerular basement membranes in diabetes cannot be conceived as a generalized alteration, but depends on the tissue examined. Furthermore, a recent study of the intimal layer of aorta from NIDDM patients showed no significant change in heparan sulphate content [37]. In this study an increased dermatan sulphate content was observed without any significant changes in other GAG. It is difficult to relate this finding to the results of our study, as the intimal and medial layers of aorta have a quite different composition including paramount differences in GAG content [38].

The changes we have found in tunica media from plaque areas comprising increased proportion of dermatan sulphate, but decreased hyaluronic acid are consistent with the findings of more recent studies of aorta from non-diabetic subjects. Many investigators have studied the GAG content of normal and atherosclerotic arteries with at times rather inconsistent findings [19, 38-42]. However, most of the more recent studies on human coronary arteries agree that dermatan sulphate increases in tunica media in atherosclerosis $[19,40]$. In the human aorta some investigators have observed increased dermatan sulphate-chondroitin sulphate [42], but others did not find any significant changes in tunica media in atherosclerosis [38]. In experimentally-induced atherosclerosis in primate aorta the most significant observation was increased dermatan sulphate [41] which is in agreement with our results. With regard to hyaluronic acid and atherosclerosis there are several reports which indicate that hyaluronic acid decreases with atherosclerotic involvement of tunica media [19,39-41].

Considering hyaluronic acid there was some similarity in our data of IDDM and NIDDM patients, but when focusing on total GAG content the results are discordant, as only NIDDM patients had a reduced GAG content. The observed difference may be related to older age and shorter diabetes duration of the NIDDM group, as these factors may be associated with a decreased GAG content.

It is remarkable that the changes we have found in normal areas of tunica media in diabetes are different from the changes observed in atherosclerotic plaques with respect to hyaluronic acid proportion. It appears that the extracellular matrix of the arterial wall in diabetes is subject to different pathological processes.
In areas with atherosclerotic plaque, a reduction in hyaluronic acid is seen and this alteration together with accumulation of dermatan sulphate is similar to that seen in non-diabetic subjects. But in areas of aorta with macroscopically normal luminal surface an increased proportion of hyaluronic acid is observed in tunica media in diabetes. In our previous studies we observed accumulation of extracellular matrix components, such as fibronectin, and type IV collagen in tunica media of aorta in diabetes independent of the presence of atherosclerotic plaques $[8,9]$. These findings together with the data of the present study strongly support the concept of a diabetic macrovascular disease which is different from classic atherosclerosis.

Acknowledgements. This work was supported by grants from the Institute of Experimental Clinical Research, University of Aarhus, the Danish Heart Foundation, and the Danish Medical Research Council.

\section{References}

1. Ledet T, Heickendorff L, Rasmussen LM (1992) Cellular mechanisms of diabetic large vessel disease. In; Alberti KGMM, DeFronzo RA, Keen H, Zimmet P (eds) International textbook of diabetes mellitus. John Wiley \& Sons Ltd, Chichester, pp 1435-1446

2. Blumenthal HT, Alez M, Goldenberg S (1960) A study of lesions of the intramural coronary artery branches in diabetes mellitus. Lab Pathol 70: 13-23

3. Ledet T (1968) Histological and histochemical changes in the coronary arteries of old diabetic patients. Diabetologia 4: 268-272

4. Ledet T (1976) Diabetic cardiopathy. Quantitative histological studies of the heart from young juvenile diabetics. Acta Path Microbiol Scand [Sect A] 84: 421-428

5. Dybdahl H, Ledet $T$ (1987) Diabetic macroangiopathy. Quantitative histopathological studies of the extramural coronary arteries from type 2 (non-insulin-dependent) diabetic patients. Diabetologia 30: $882-886$

6. Ferrier TM (1964) Radiological demonstrable arterial calcifications in diabetes mellitus. Aust Ann Med 13:222-228

7. Neubauer B (1971) A quantitative study of peripheral arterial calcification and glucose tolerance in elderly diabetics and nondiabetics. Diabetologia 7: 409-416

8. Rasmussen LM, Heickendorff L (1989) Accumulation of fibronectin in aortas from diabetic patients. A quantitative immunohistochemical and biochemical study. Lab Invest 61: 440-446

9. Rasmussen LM, Ledet T (1993) Aortic collagen alterations in human diabetes mellitus. Changes in basement membrane collagen content and in the susceptibility of total collagen to cyanogen bromide solubilisation. Diabetologia 36: 445-453

10. Oxlund H, Rasmussen LM, Andreassen TT, Heickendorff L (1989) Increased aortic stiffness in patients with type 1 (insulin-dependent) diabetes mellitus. Diabetologia 32: 748 752

11. Ichida T, Kalant N (1968) Aortic glycosaminoglycans in atheroma and alloxan diabetes. Can J Biochem 46: 249-260

12. Malathy K, Kurup PA (1972) Metabolism of glycosaminoglycans in alloxan diabetic rats. Changes in tissue glycosaminoglycans. Diabetes 21:1162-1167 
13. Sirek OV, Sirek A, Cuckerman E (1981) Intermittent hyperinsulinaemia and arterial glycosaminoglycans in dogs. Diabetologia 21: 154-159

14. Wight TN (1989) Cell biology of arterial proteoglycans. Arteriosclerosis 9: 1-20

15. Iverius PH (1972) The interaction between human plasma lipoproteins and connective tissue glycosaminoglycans. J Biol Chem 247: 2607-2613

16. Camejo G, Rosengren B, Olzon U et al. (1990) Molecular basis for the association of proteoglycans with low density lipoproteins: its effect on the structure of the lipoprotein particle. Eur Heart J 11 [Suppl E]: 164-173

17. Vijayagopal $P$, Radhakrishnamurthy $B$, Srinivasan $S R$, Berenson GS (1980) Studies of biological properties of proteoglycans from bovine aorta. Lab Invest 42: 190-196

18. Fritze LMS, Reilly CF, Rosenberg RD (1985) An antiproliferative heparan sulphate species produced by postconfluent smooth muscle cells. J Cell Biol 100: 1041-1049

19. Ylä-Herttuala S, Sumuvuori H, Karkola K, Möttönen M, Nikkari T (1986) Glycosaminoglycans in normal and atherosclerotic human coronary arteries. Lab Invest 54: 402-407

20. Blumenkrantz N, Asboe-Hansen G (1973) New method for quantitative determination of uronic acids. Anal Biochem 54: 484-489

21. Boas NF (1953) Method for determination of hexosamines in tissues. J Biol Chem 204: 553-563

22. Wight TN, Curwen KD, Litrenta MM, Alonso DR, Minick CR (1983) Effect on endothelium on glycosaminoglycan accumulation in injured rabbit aorta. Am J Pathol 113:156-164

23. Cappelletti R, Del Rosso M, Chiarugi VP (1975) A new electrophoretic method for the complete separation of all known animal glycosaminoglycans in a monodimensional run. Anal Biochem 99: 311-315

24. Ross R (1971) The smooth muscle cells: II. Growth of smooth muscle cells in culture and formation of elastic fibres. J Cell Biol 50: 172-186

25. Järveläinen H, Rönnemaa T, Lethonen A (1987) Effect of sera from male type 1 (insulin-dependent) diabetics on human aortic smooth muscle cells in culture. Acta Endocrinol 114: 362-370

26. Järveläinen H, Rönnemaa T, Viikari J (1986) Effect of sera from male type 2 (non-insulin-dependent) diabetics on human aortic smooth muscle cells in culture. Med Biol 64: 361-366

27. Baandrup U, Ledet T, Rasch R (1981) Experimental diabetic cardiopathy preventable by insulin treatment. Lab Invest 45: 169-173

28. Ledet T, Fischer-Dzoga K, Wissler RW (1976) Growth of rabbit aortic smooth-muscle cell cultures in media containing diabetic and hyperlipemic serum. Diabetes 25: 207-215
29. Hollman J, Thiel J, Schmidt A, Buddecke E (1986) Increased activities of chondroitin sulfate synthesizing enzymes during proliferation of arterial smooth muscle cells. Exp Cell Res 167: 484-494

30. Deudon E, Berrou E, Breton M, Picard J (1992) Growth related production of proteoglycans and hyaluronic acid in synchronous arterial smooth muscle cells. Int J Biochem 24: $465-470$

31. Hronowski L, Anastassiades TP (1980) The effect of cell density on net rates of glycosaminoglycan synthesis and secretion by cultured rat fibroblasts. J Biol Chem 255: 1009110099

32. Bachem MG, Riess U, Melchoir R, Sell KM, Gressner AM (1989) Transforming growth factors (TGF $\alpha$ and TGF $\beta 1$ ) stimulate chondroitin sulfate and hyaluronate synthesis in cultured rat liver fat storing cells. FEBS Lett 257: 134-137

33. Heldin P, Laurent TC, Heldin C-H (1989) Effect of growth factors on hyaluronan synthesis in cultured human fibroblasts. Biochem J 258: 919-922

34. Cohen MP, Foglia VG (1970) Aortic mucopolysaccharides in experimental diabetes. Diabetes 19:639-643

35. Parthasarathy N, Spiro RG (1982) Effect of diabetes on the glycosaminoglycan component of the human glomerular basement membrane. Diabetes 31: 738-741

36. Shimorura H, Spiro RG (1987) Studies on macromolecular components of human glomerular basement membrane and alterations in diabetes. Diabetes 36: 374-381

37. Wasty F, Alavi MZ, Moore S (1993) Distribution of glycosaminoglycans in the intima of human aortas: changes in atherosclerosis and diabetes mellitus. Diabetologia 36: 316322

38. Stevens RL, Colombo M, Gonzales JJ, Hollander W, Schmid K (1976) The glycosaminoglycans of the human artery and their changes in atherosclerosis. J Clin Invest 58: 470-481

39. Klynstra FB, Böttcher CJF, Van Der Laan EJ (1967) Distribution and composition of acid mucopolysaccharides in normal and atherosclerotic human aortas. J Atheroscler Res 7: 301-309

40. Tammi M, Sepälä PO, Lehtonen A, Möttönen M(1978) Connective tissue components in normal and atherosclerotic human coronary arteries. Atherosclerosis 29: 191-194

41. Wagner WD, Salisbury BGJ (1978) Aortic total glycosaminoglycan and dermatan sulfate changes in atherosclerotic rhesus monkeys. Lab Invest 39: 322-328

42. Hollmann J, Schmidt A, von Bassewitz D-B, Buddecke E (1989) Relationship of sulphated glycosaminoglycans and cholesterol content in normal and atherosclerotic human aorta. Arteriosclerosis 9: $154-\mathbf{1 5 8}$ 